\title{
Evolución de lesiones oculares en el trabajo en las comunidades autónomas españolas en el periodo 2008-2018
}

\section{Trends in work-related eye injuries in Spanish Autonomous Communities during 2008-2018}

\author{
Sergio Martín Prieto ${ }^{1}$ (D) 0000-0002-9802-623 \\ Cristina Alvarez Peregrina ${ }^{1}$ (1) 0000-0003-1097-4581 \\ Israel Thuissard Vasallo' (i) 0000-0002-6990-0619 \\ Carlos Catalina Romero² (i) 0000-0003-1322-9165 \\ Eva Calvo Bonacho ${ }^{2}$ \\ César Villa Collar ${ }^{1}$ (1) 0000-0002-6743-8264 \\ Miguel Ángel Sánchez Tena' [D 0000-0002-2583-1789
}

${ }^{1}$ Escuela de Biomedicina y Ciencias de la Salud, Universidad Europea de Madrid, Madrid, España. ${ }^{2}$ Ibermutua (Mutua colaboradora con la seguridad social n² 274), Madrid, España.

Fechas · Dates

Recibido: 2020.02 .18 Aceptado: 2021.02 .08

Publicado: 2021.04.21
Correspondencia · Corresponding Author

Sergio Martín Prieto

C/ Fuenlabrada 3 Bajo D. 28019 Madrid

martin.prieto.sergio@gmail.com 


\title{
Resumen
}

Objetivos: Analizar las diferencias en la evolución de las lesiones oculares en el trabajo entre las comunidades autónomas en España.

Métodos: Se llevó a cabo un estudio descriptivo, retrospectivo y longitudinal de los accidentes laborales y las enfermedades profesionales de una Mutua Colaboradora de la Seguridad Social entre las diferentes comunidades autónomas españolas en el intervalo de tiempo de 2008-2018. Se analizaron aquellos accidentes que provocaron lesión en alguna estructura ocular, comparándose la incidencia por cada 100000 asegurados y el riesgo relativo (RR) de sufrir una lesión ocular en el trabajo.

Resultados: Se observó una disminución en la evolución de la incidencia de lesiones oculares en el trabajo en todas las comunidades, aunque con dos periodos diferenciados. El porcentaje de cambio anual disminuye en todas las comunidades hasta el 2013, pero entre 2013-2018 esta tendencia sólo se mantiene en Asturias, Castilla-La Mancha, Islas Canarias, Madrid, Murcia y Navarra. Castilla- La Mancha fue la comunidad autónoma con mayor incidencia (886.56/100 000) y riesgo relativo de sufrir una lesión ocular (RR 2.66; IC 95\% 2.58 - 2.75), mientras Cataluña presentó la menor incidencia (82.25) y riesgo relativo (RR 0.25 ; IC $95 \% 0.22-0.28$ ).

Conclusiones: Durante el periodo 2008-2018 se produce una disminución en la incidencia de lesiones oculares en el trabajo en todas las comunidades autónomas con un cambio de tendencia a partir del año 2013.

Palabras clave: Accidentes de trabajo; Lesiones oculares; Traumatismos ocupacionales; Prevención de accidentes; Seguro de accidentes; Enfermedades profesionales.

\begin{abstract}
Objectives: To analyze the differences in work-related eye injury trends among the different Autonomous Communities in Spain.

Methods: This was a descriptive, retrospective and longitudinal study based on a Social Security-affiliated mutual insurance company work injury and illness database for the period 2008-2018 that included all Spanish Autonomous Communities. Accidents that caused an injury to any ocular structure were analysed, and both the incidence of work-related eye-injuries per 100000 insured workers and the relative risk of suffering an eye injury were compared.
\end{abstract}

Results: We observed a general decrease in work-related eye injuries in all of the communities, although with two distinct time periods. The annual percentage change decrease occurred in all communities until 2013. From 2013 to 2018, however, this decreasing trend was only observed in Asturias, Castilla-La Mancha, Canary Islands, Madrid, Murcia and Navarra. Castilla-La Mancha was the Autonomous Community with the highest incidence (886.56/100.000), as well as the highest relative risk of suffering an eye injury (RR 2.66; IC $95 \% 2.58$ - 2.75). At the other end, the lowest incidence (82.25/100.000) and RR (RR 0.247; IC $95 \% 0.22-0.28$ ) were observed in Catalonia. 
Conclusions: We observed a statistically significance decrease in the incidence and relative risk of work-related eye injuries in all Autonomous Communities over the study period, although this trend changed from 2013 onward.

Keywords: occupational accidents; eye injuries; occupational injuries; accident prevention; accident insurance; professional sickness.

\section{Introducción}

Según las estadísticas de accidentes laborales con baja en España, las estructuras oculares supusieron alrededor del 3\% de todas las bajas laborales en 2018, siendo la estructura más afectada de la cabeza(1). El porcentaje de lesiones oculares asociadas al trabajo alrededor del mundo se sitúa entre el $25.40 \%$ y el $57.12 \%(2,3)$, siendo el principal origen de las lesiones oculares que acuden a urgencias en distintas regiones de Asia (46.50\% en Chaosang, China(4); 38.90\% en Kaohsiung, Taiwan(5); 42.90\% en Chongqing, China(6) y Europa (57.12\% en Torino, Italia(3); 34\% en Helsinki, Finlandia ${ }^{(7)}$. El trabajo más reciente llevado a cabo en España por Serrano et al. en 2008, señaló que el $25.20 \%$ de las lesiones oculares tienen origen laboral(8). Otro estudio local Ilevado a cabo en Baleares en los años 2005 y 2006, mostró que las lesiones oculares en el trabajo afectaron al $0.84 \%$ del total de asegurados de una mutua laboral(9)

La incidencia de accidentes laborales varía a lo largo del tiempo y se ha correlacionado con la evolución del mercado laboral(10). Así, en España se observó una disminución progresiva de la incidencia por cada 100000 trabajadores entre los años 2007 y 2012, sin embargo, a partir de ese año la tendencia se invierte ${ }^{(10)}$, pasando de 2948.9 en 2012 hasta 3325.9 en 2015(11).

Sin embargo, este aumento se produjo de manera asimétrica en las distintas comunidades autónomas (CC. AA.). En el periodo 2012-2018 el mayor aumento se produjo en Navarra (+31.50\%), Baleares (+27.00\%) y Extremadura (+26.30\%). En el País Vasco, apenas existió variación (+0.40\%), e incluso en otras CC. AA. se produjo una reducción en la incidencia: Asturias (-3.70\%) y la Ciudad Autónoma de Melilla $(-7.20 \%)^{(11)}$.

El objetivo principal de este estudio es analizar las diferencias en la evolución de las lesiones oculares en el trabajo entre las distintas CC. AA. en España en el periodo $2008-2018$.

\section{Métodos}

Se realizó un estudio descriptivo, retrospectivo y longitudinal donde se analizaron los accidentes oculares que ocasionaron una lesión en trabajadores cuyas empresas están asociadas a la Mutua Colaboradora de la Seguridad Social n²74. Cuando un trabajador sufre un accidente laboral, acude a los servicios médicos de la Mutua para una evaluación completa de su lesión y las consecuencias de esta, 
recogiéndose los datos personales del sujeto y el tipo de lesión que se produce. Se denominaron a este tipo de lesiones oculares como lesiones oculares en el trabajo (LOT) en las que se englobaron también las enfermedades oculares profesionales.

Se evaluaron LOT durante un periodo comprendido entre el 1 de enero de 2008 y el 31 de diciembre de 2018. El área de estudio comprendió todas las regiones de España incluyendo Ceuta y Melilla con una población de 46650300 habitantes según el último censo de 2018, de ellos, el 48.87\% se encontraban en edad laboral(12). Las LOT consideradas fueron las que tuvo la Mutua a fecha 31 de diciembre del año de estudio y se compararon con el número de asegurados que tuvo la Mutua en la misma fecha.

La investigación se ha realizado siguiendo los principios de la Declaración de HeIsinki y fue aprobada por el comité de ética de la investigación de la Universidad Europea de Madrid con el código CIPI/19/03. Todos los datos proporcionados por la Mutua fueron anónimos.

\section{Criterios de inclusión}

Se consideraron LOT todas aquellas lesiones oculares provocadas por accidentes y/o enfermedades profesionales, según lo establecido por el Artículo 157 del Real Decreto Legislativo 8/2015 de 30 de octubre de la Ley General de la Seguridad Social, que sufre un trabajador en su puesto de trabajo o in itinere y que afecta a cualquier estructura ocular. Estas lesiones fueron clasificadas por personal médico cualificado atendiendo a la clasificación internacional oficial CIE-9-MC(13) y correlacionada con la clasificación ICD-10(14). Las LOT incluidas en el estudio son aquellas que se encuentran entre los códigos numéricos de la clasificación CIE9-MC 360 y 379: "Trastornos del ojo y los anexos". Se ha considerado cada lesión como una unidad de estudio.

Para clasificar los casos en las distintas CC. AA., se utilizaron los 2 primeros números del código postal provincial del domicilio de la base de datos de la Mutua, asociándolo a la comunidad correspondiente.

\section{Análisis estadístico}

Se han calculado las tasas de incidencia de LOT por cada 100000 asegurados con el objetivo de tener una medida homogénea para todas las CC. AA. y poder así compararlas a lo largo del periodo de estudio. Así mismo, se ha calculado el riesgo relativo (RR) tomando la incidencia media en España como referencia para comprobar en cuál de ellas existe un mayor riesgo de sufrir una LOT.

Se estableció un porcentaje de cambio anual (PCA) mediante una regresión binomial negativa para estudiar la evolución de la incidencia durante el periodo de estudio. 
Se ha aplicado la prueba Chi-cuadrado para analizar la existencia de diferencias estadísticamente significativas entre los resultados obtenidos entre las distintas comunidades y sobre los distintos periodos de tiempo analizado.

Para la realización del análisis estadístico se ha utilizado el paquete SPSS, v. 21.0 (IBM Corp; Armonk, NY; USA), considerándose la existencia de diferencias estadísticamente significativas cuando el p-valor sea inferior al 0.05 .

\section{Resultados}

Se analizaron un total de 11695598 asegurados por la Mutua durante el periodo de 2008 a 2018. Se tuvieron en cuenta todas las CC. AA. más las ciudades autónomas de Ceuta y Melilla. En la Tabla 1 se puede observar la distribución de LOT y de asegurados en las distintas zonas analizadas. Del total de LOT registrados por la Mutua, las CC. AA. con más casos fueron Madrid, Murcia, Castilla y León y Andalucía, siendo Ceuta y Melilla las regiones con menos casos. Asimismo, Madrid, Murcia, Andalucía, Comunidad Valenciana y Castilla y León representan, por este orden, las CC. AA. con mayor porcentaje de asegurados del total de asegurados de la Mutua.

Tabla 1: Distribución de lesiones oculares en el trabajo y de asegurados de la Mutua según las distintas comunidades y ciudades autónomas (entre 2008 y 2018).

\begin{tabular}{lcccc}
\hline Comunidades Autónomas & Lesiones oculares en el trabajo & \multicolumn{2}{c}{ Asegurados } \\
\hline Andalucía & $\mathbf{n}$ & $\%^{\mathbf{a}}$ & $\mathbf{n}$ & $\mathbf{\%}^{\mathbf{b}}$ \\
Aragón & 5853 & 11.65 & 1703751 & 14.57 \\
Asturias & 73 & 0.15 & 46836 & 0.40 \\
Cantabria & 3838 & 7.64 & 907820 & 7.79 \\
Castilla-La Mancha & 154 & 0.31 & 50510 & 0.43 \\
Castillay León & 4003 & 7.97 & 451522 & 3.86 \\
Cataluña & 6685 & 13.31 & 1792185 & 10.19 \\
Ceuta & 274 & 0.55 & 333141 & 2.85 \\
Comunidad Valenciana & 4 & 0.00 & 2941 & 0.02 \\
Extremadura & 4467 & 8.90 & 1505820 & 12.88 \\
Galicia & 589 & 1.17 & 182003 & 1.56 \\
Islas Baleares & 2198 & 4.38 & 301288 & 2.58 \\
Islas Canarias & 272 & 0.54 & 83613 & 0.71 \\
La Rioja & 433 & 0.86 & 145393 & 1.24 \\
Madrid & 117 & 0.22 & 38399 & 0.33 \\
Melilla & 13224 & 26.32 & 2515450 & 21.51 \\
Murcia & 2 & 0.00 & 2642 & 0.02 \\
Navarra & 7605 & 15.14 & 2118630 & 18.17 \\
País Vasco & 128 & 0.25 & 28279 & 0.24 \\
ESPAÑA & 321 & 0.64 & 85375 & 0.73 \\
\hline Porcenta & 234 & & 11695598 & \\
\hline
\end{tabular}

aPorcentaje calculado sobre el total de LOT en la Mutua en toda España.

b Porcentaje calculado sobre el total de asegurados de la Mutua en toda España. 


\section{Incidencia de LOT por cada 100000 asegurados y evolución du- rante el periodo de estudio}

Se decidió excluir de este análisis a Ceuta y Melilla, debido al escaso número de LOT (4 en Ceuta y 2 en Melilla, Tabla 1) y la imposibilidad de comparar estos valores a lo largo del tiempo.

La incidencia total acumulada en España durante el periodo de estudio fue de 429.75 LOT por cada 100000 asegurados, existiendo una gran variabilidad entre las distintas CC. AA. En la Figura 1 se puede observar que sólo Castilla-La Mancha (886.56), Galicia (729.53), Castilla y León (560.74), Madrid (525.71) y Navarra (452.63) tienen una incidencia mayor a la media española. Mientras que, la comunidad con menor incidencia total acumulada durante todo el periodo es Cataluña con 82.25 casos por 100000 asegurados, seguida de Aragón con 155.86 y La Rioja con 289.07.

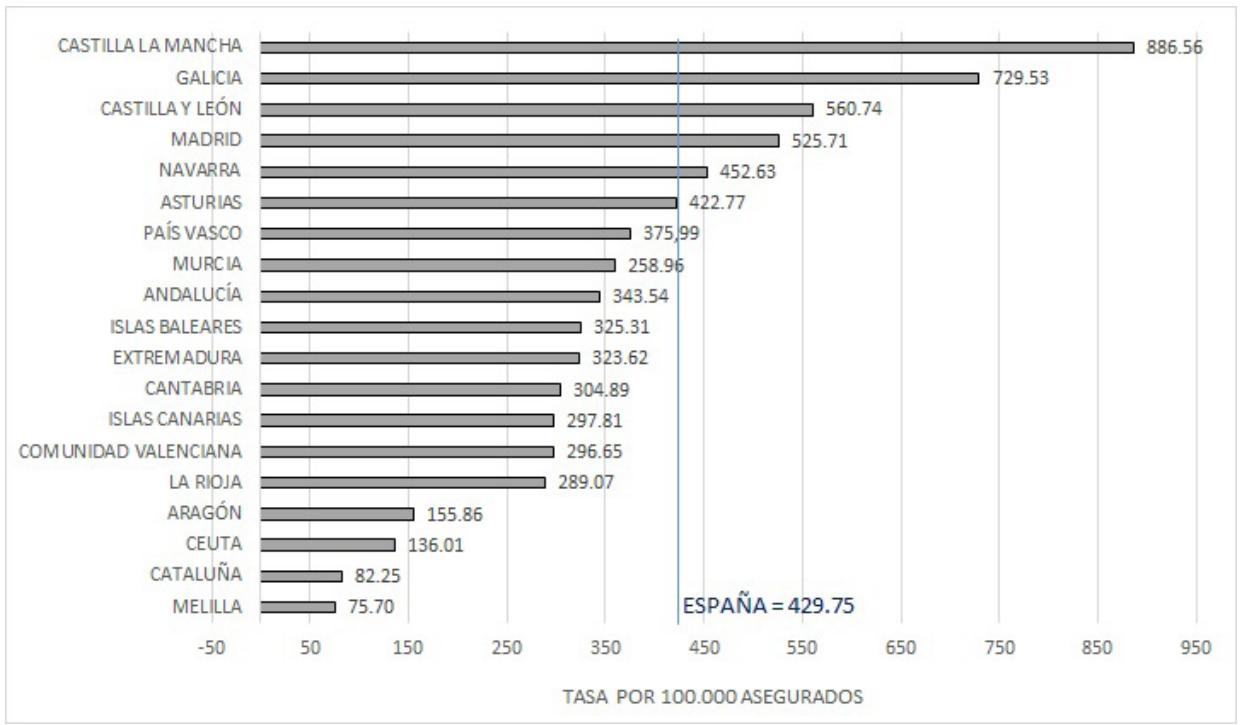

Figura 1: Incidencia total acumulada de lesiones oculares en el trabajo entre 2008 y 2018.

Si se analiza la evolución de la incidencia de LOT por cada 100000 asegurados durante todo el periodo de estudio, se observa una disminución en todas las CC. AA. reflejada en la pendiente negativa de las curvas (Figura 2).

Cuando se evalúa la incidencia de LOT al comienzo (2008) y al final del estudio (2018) en cada una de las CC. AA., se puede observar que existe una disminución estadísticamente significativa entre ambos valores en todas ellas. Sin embargo, existe un punto de inflexión en el año 2013 cuando la incidencia ralentiza su disminución o directamente la evolución de la incidencia a partir de ese año cambia de tendencia. En la Tabla 2 se puede observar como el PCA disminuye de manera estadísticamente significativa en todas las CC. AA. excepto en Aragón, Navarra y el País Vasco en el periodo 2008-2013. Sin embargo, en el periodo 2013-2018, el 
PCA negativo sólo se observa en Asturias (PCA -0.30; IC 95\% -11.90 a 12.90), Castilla-La Mancha (PCA -1.90; IC 95\% -12.90 a 10.40), Islas Canarias (PCA -8.70; IC 95\% -31.90 a 22.30), Madrid (PCA -6.60; IC 95\% -13.60 a 1.00), Murcia (PCA -0.60; IC $95 \%-9.70$ a 9.50) y Navarra (PCA -3.00; IC 95\% -32.10 a 38.60), no siendo esta disminución estadísticamente significativa en ninguna de ellas.

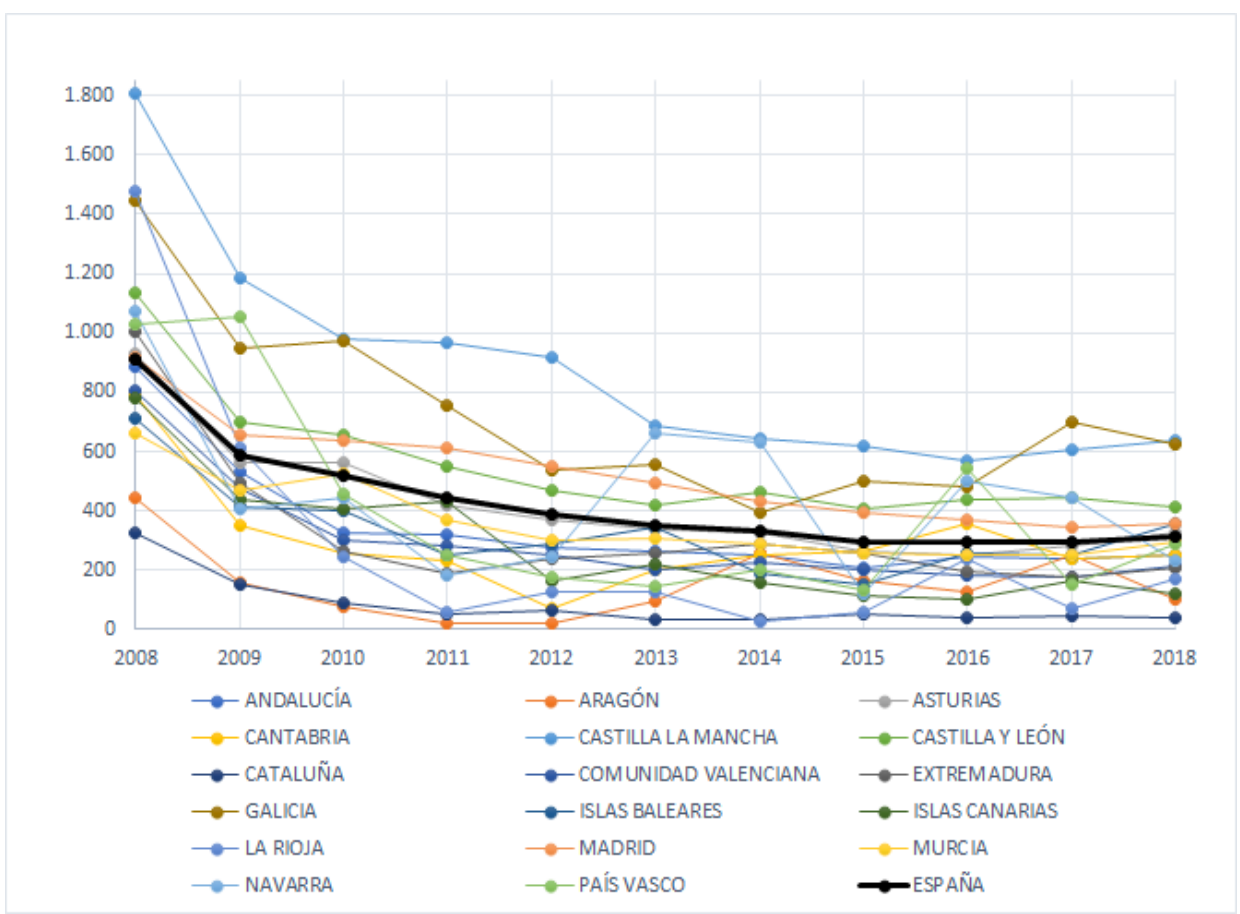

Figura 2: Evolución de la incidencia de lesiones oculares en el trabajo por cada 100000 asegurados entre 2008 y 2018.

\section{Riesgo relativo de sufrir una LOT}

El mayor riesgo de sufrir LOT se da en Castilla-La Mancha (RR 2.66; IC 95\% 2.58 - 2.75) y en Galicia (RR 2.19; IC 95\% 2.10 - 2.23), y el menor riesgo se da en Cataluña (RR 0.25; IC 95\% 0.22 - 0.28) y Aragón (RR 0.47; IC 95\% 0.37 - 0.59) (Tabla 3). 
Tabla 2: Incidencia de lesiones oculares en el trabajo por cada 100000 asegurados en 2008, 2013 y 2018, y porcentaje de cambio anual diferenciado en los periodos 2008-213 y 2013-2018.

\section{8-2013}

\begin{tabular}{|c|c|c|c|c|c|c|c|}
\hline CC. AA. ${ }^{a}$ & 2008 & 2013 & 2018 & PCA & IC (95\%) & PCA & IC (95\%) \\
\hline Andalucía & 886.54 & 263.68 & 251.70 & $-22.70^{*}$ & $\begin{array}{l}-30.70 a \\
-13.80\end{array}$ & 3.60 & $\begin{array}{l}-10.00 \mathrm{a} \\
19.30\end{array}$ \\
\hline Aragón & 445.32 & 99.06 & 99.70 & -16.70 & $\begin{array}{l}-43.10 a \\
21.90\end{array}$ & 8.70 & $\begin{array}{l}-14.00 a \\
37.50\end{array}$ \\
\hline Asturias & 932.53 & 338.19 & 324.34 & $-18.80^{*}$ & $\begin{array}{l}-26.10 \mathrm{a} \\
-10.90\end{array}$ & -0.30 & $\begin{array}{l}-11.90 \mathrm{a} \\
12.90\end{array}$ \\
\hline Cantabria & 791.14 & 201.16 & 252.74 & $-23.30 *$ & $\begin{array}{l}-36.50 \mathrm{a} \\
-7.30\end{array}$ & 14.40 & $\begin{array}{l}-5.90 a \\
39.20\end{array}$ \\
\hline $\begin{array}{l}\text { Castilla-La } \\
\text { Mancha }\end{array}$ & 1805.05 & 685.29 & 634.49 & $-16.60^{*}$ & $\begin{array}{l}-23.40 \mathrm{a} \\
-9.10\end{array}$ & -1.90 & $\begin{array}{l}-12.90 \mathrm{a} \\
10.40\end{array}$ \\
\hline Castilla y León & 1134.79 & 417.11 & 410.45 & $-16.90 *$ & $\begin{array}{l}-22.90 \text { a } \\
-10.50\end{array}$ & 1.90 & $\begin{array}{l}-7.40 a \\
12.10\end{array}$ \\
\hline Cataluña & 327.24 & 36.49 & 41.42 & $-35.10^{\star}$ & $\begin{array}{l}-45.10 \text { a } \\
-23.30\end{array}$ & 8.40 & $\begin{array}{l}-17.50 a \\
42.30\end{array}$ \\
\hline $\begin{array}{l}\text { Comunidad } \\
\text { Valenciana }\end{array}$ & 805.27 & 200.78 & 215.05 & $-23.80 *$ & $\begin{array}{l}-31.90 a \\
-14.80\end{array}$ & 2.50 & $\begin{array}{l}-10.70 a \\
17.70\end{array}$ \\
\hline Extremadura & 1001.66 & 255.36 & 624.19 & $-24.40 *$ & $\begin{array}{l}-36.40 a \\
-10.20\end{array}$ & 1.50 & $\begin{array}{l}-18.60 a \\
26.50\end{array}$ \\
\hline Galicia & 1444.81 & 557.88 & 624.19 & $-19.70 *$ & $\begin{array}{l}-26.30 \mathrm{a} \\
-12.40\end{array}$ & 6.60 & $\begin{array}{l}-4.90 \mathrm{a} \\
19.50\end{array}$ \\
\hline Islas Baleares & 715.10 & 345.26 & 454.75 & $-18.90^{*}$ & $\begin{array}{l}-28.50 a \\
-7.90\end{array}$ & 6.60 & $\begin{array}{l}-8.20 a \\
23.80\end{array}$ \\
\hline Islas Canarias & 779.69 & 221.10 & 120.93 & $-23.70 *$ & $\begin{array}{l}-35.40 \mathrm{a} \\
-9.90\end{array}$ & -8.70 & $\begin{array}{l}-31.90 a \\
22.30\end{array}$ \\
\hline La Rioja & 1475.69 & 125.71 & 170.82 & $-45.80^{*}$ & $\begin{array}{l}-61.30 \text { a } \\
-24.10\end{array}$ & 26.20 & $\begin{array}{l}-31.00 \mathrm{a} \\
130.60\end{array}$ \\
\hline Madrid & 918.88 & 495.90 & 358.43 & $-11.10^{*}$ & $\begin{array}{l}-16.20 a \\
-5.80\end{array}$ & -6.60 & $\begin{array}{l}-13.60 \mathrm{a} \\
1.00\end{array}$ \\
\hline Murcia & 661.27 & 307.82 & 296.30 & $-15.30 *$ & $\begin{array}{l}-22.00 a \\
-8.10\end{array}$ & -0.60 & $\begin{array}{l}-9.70 a \\
9.50\end{array}$ \\
\hline Navarra & 1074.19 & 661.08 & 233.75 & -10.60 & $\begin{array}{l}-33.90 \mathrm{a} \\
21.0\end{array}$ & -3.00 & $\begin{array}{l}-32.10 a \\
38.60\end{array}$ \\
\hline País Vasco & 1026.60 & 145.18 & 290.26 & -32.30 & $\begin{array}{l}-54.20 \mathrm{a} \\
0.20\end{array}$ & 19.30 & $\begin{array}{l}-25.70 a \\
91.40\end{array}$ \\
\hline España & 912.48 & 353.1 & 314.72 & $-17.40^{*}$ & $\begin{array}{l}-22.70 a \\
-11.80\end{array}$ & -1.10 & $\begin{array}{l}-9.20 a \\
8.00\end{array}$ \\
\hline
\end{tabular}

${ }^{\star} \mathrm{p}<0.05$

CC. AA., Comunidades Autónomas; PCA, Porcentaje de cambio anual; IC, Intervalo de confianza. 
Tabla 3: Asociación entre las lesiones oculares en el trabajo y las comunidades autónomas (entre 2008 y 2018).

\begin{tabular}{lll}
\hline Comunidades Autónomas & $\begin{array}{l}\text { Riesgo relativo (Intervalo de confianza } \\
\text { 95\%) }\end{array}$ & p-valor \\
\hline Cataluña & $0.20(0.22-0.28)$ & $<0.001$ \\
Aragón & $0.47(0.37-0.59)$ & $<0.001$ \\
La Rioja & $0.87(0.72-1.05)$ & 0.136 \\
Comunidad Valenciana & $0.89(0.81-0.98)$ & $<0.001$ \\
Islas Canarias & $0.89(0.81-0.98)$ & 0.021 \\
Cantabria & $0.92(0.78-1.07$ & 0.275 \\
Extremadura & $0.97(0.90-1.05)$ & 0.494 \\
Islas Baleares & $0.98(0.87-1.10)$ & 0.703 \\
Andalucía & $1.03(1.00-1.06)$ & 0.032 \\
Murcia & $1.08(1.05-1.11)$ & $<0.001$ \\
País Vasco & $1.13(1.01-1.26)$ & 0.030 \\
Asturias & $1.27(1.23-1.31)$ & $<0.001$ \\
Navarra & $1.36(1.14-1.62)$ & $<0.001$ \\
Madrid & $1.58(1.55-1.61)$ & $<0.001$ \\
Castilla y León & $1.68(1.64-1.73)$ & $<0.001$ \\
Galicia & $2.19(2.10-2.23)$ & $<0.001$ \\
Castilla-La Mancha & $2.66(2.58-2.75)$ & $<0.001$ \\
España & Referencia & \\
\hline
\end{tabular}

\section{Discusión}

Castilla-La Mancha y Cataluña fueron las CC. AA. con mayor y menor incidencia y RR, respectivamente, de sufrir una LOT. La evolución de la incidencia entre el comienzo (2008) y el final (2018) del periodo de estudio, muestra una disminución estadísticamente significativa en todas las CC. AA., aunque con 2 periodos claramente diferenciados: el primero que engloba 2008-2013, donde se observa una disminución en todas las regiones, y un segundo periodo entre 2013-2018 donde se observa gran variabilidad entre CC. AA.

En el análisis de los resultados de las distintas CC. AA., se observó en todas ellas excepto en Castilla-La Mancha (0.89\%), un porcentaje de incidencia de LOT menor que el obtenido en el estudio llevado a cabo por Gómez y Lavaria ${ }^{(9)}$ en Palma de Mallorca y Manacor (0.84\%). La incidencia en estas 2 localidades es también muy superior a la encontrada en nuestro estudio para toda España (0.43\%). Esta diferencia puede deberse a que el estudio de Gómez y Lavaria es anterior (2005-2006) y menos representativo, ya que sólo analizó dos pequeñas localidades.

En otros estudios, se ha observado que la incidencia de LOT puede variar entre 88 y 1920 accidentes por cada 100000 personas ${ }^{(7,15)}$. En el presente estudio, todas 
las CC. AA. presentaron incidencias entre esos valores a excepción de Cataluña (82.25 LOT por 100000 asegurados). Sin embargo, estos datos son difícilmente comparables, ya que los casos contemplados en dichos estudios no hacen referencia sólo a grupos de población trabajadora, sino a sujetos que acudieron a su hospital de referencia. Además, las lesiones analizadas en muchos de ellos fueron únicamente traumas oculares, mientras que en el presente estudio se analizaron todo tipo de lesiones.

Se observó una disminución en la incidencia comparando los años 2008 y 2018 (principio y final del periodo de estudio), donde estas diferencias son estadísticamente significativas en todas las CC. AA. Esto puede deberse a varias razones, la primera de ellas es la disminución de trabajadores como consecuencia del aumento del paro(12) lo que provocó una disminución del número de accidentes y lesiones asociadas. En segundo lugar, los cambios en el sector laboral predominante al que se dedicaron los trabajadores. En un estudio previo llevado a cabo por este grupo, la mayor incidencia de LOT en todo el periodo se produce en el sector industria con 1538.17 y la construcción con 1381.52 LOT por cada 100000 asegurados $^{(16)}$. En estos 2 sectores hay una disminución en el número de trabajadores en la Mutua desde el $27.59 \%$ de asegurados en 2008 al 18.92\% en 2018, lo que provocó una disminución estadísticamente significativa $(p<0.05)$ de la incidencia de LOT entre 2008 y 2018 en estos 2 sectores en todas las CC. AA. La fuerte influencia de estos sectores también se observa si se analizan los datos oficiales de accidentes con baja que afecte a cualquier estructura corporal en toda España; menos de la mitad de accidentes se producen en el sector industria y construcción en el año 2018 con respecto a $2008^{(1,17)}$.

Sin embargo, esta tendencia descendente en la incidencia de LOT se interrumpió a partir del año 2013. En el periodo 2008-2013 se observó una disminución del PCA en todas las CC. AA. siendo estadísticamente significativa $(p<0.05)$ en todas ellas menos en Aragón, Navarra y País Vasco. Por el contrario, en el periodo 2013-2018, la tendencia se invirtió, observándose únicamente un PCA negativo en 6 regiones de España: Asturias, Castilla-La Mancha, Islas Canarias, Madrid, Murcia y Navarra. Esta variación en la tendencia descendente de las LOT en varias CC. AA. en España, podría deberse al aumento en la precariedad asociada a la implantación de la nueva reforma laboral en el año 2012(11).

Castilla-La Mancha es la comunidad autónoma que mayor RR tiene de sufrir un LOT, sin embargo, desde el año 2008 han sido varios los programas de prevención de riesgos laborales que han puesto en marcha. A pesar de esto, la propia administración pública de esta comunidad, reconoció en el año 2017, que se han detectado ineficacias en la puesta en marcha de estas políticas y que para paliarlas sería necesario poner en relación la formación preventiva enmarcada en el ámbito educativo y de formación profesional(18). Por el contrario, parece que las políticas puestas en marcha por la Generalitat de Cataluña a partir del año 2005 en materia de prevención de riesgos laborales, han sido más efectivas en su aplicación y puede ser una de las causas para que sea ésta la comunidad autónoma con menor RR de sufrir un $\mathrm{LOT}^{(19)}$. 
La principal limitación del presente estudio es la imposibilidad de conocer los mecanismos por los que se han producido cada una de las lesiones, por lo que no se ha podido profundizar en las razones de las diferencias observadas entre las distintas CC. AA. Conociendo los resultados del presente estudio, sería conveniente en próximas investigaciones escoger un número menor pero representativo de casos en las distintas regiones y analizar sus historias clínicas. De esta manera, se conocerían los mecanismos exactos por los que se producen las LOT, lo que permitiría desarrollar planes más específicos de prevención y protección en las empresas de cada CC. AA.

La disminución de la incidencia y RR de LOT parece estar directamente relacionada con las características socioeconómicas y laborales del periodo de estudio en toda España, caracterizado por una fuerte crisis económica. Esta crisis redujo en número de trabajadores y, como consecuencia, el número de LOT en el sector industria y construcción ${ }^{(20)}$. El cambio de tendencia a partir del año 2013 muestra los efectos de la nueva política laboral estatal establecida en 2012, aunque la evolución asimétrica entre las CC. AA. entre 2013-2018 señala la importancia de las políticas de prevención que pueden establecer las distintas regiones españolas.

El presente estudio es el que engloba el mayor número de casos y área de estudio de los que tenemos constancia en Europa, lo que puede ser una buena base para poder desarrollar planes más amplios de prevención. Además, es el primer estudio que analiza las diferencias en la incidencia de LOT entre distintas regiones de España. El conocimiento por parte de las distintas administraciones públicas de datos epidemiológicos permite a estas comprobar donde pueden mejorar sus políticas de prevención. Igualmente, la comparación con otras comunidades puede conllevar el intercambio de información para disminuir, en este caso, la incidencia de LOT en función de dichas políticas. Teniendo en cuenta estas diferencias entre las distintas CC. AA. y la facilidad de protección que presentan las distintas estructuras oculares, este estudio epidemiológico puede ser un punto de partida para plantear políticas estatales más ambiciosas en prevención de LOT.

\section{Financiación}

Los autores declaran que no han recibido financiación para la autoría y/o publicación de este artículo.

\section{Conflicto de intereses}

Los autores declaran que no existen conflictos de intereses en la autoría y/o publicación de este artículo.

\section{Bibliografía}

1. Accidentes de trabajo con baja y recaídas. Principales resultados. Estadísticas de accidentes de trabajo 2018 [página principal en internet]. Madrid: Ministerio de Trabajo Migraciones y Seguridad social. [actualizado 14 Oct 2019; citado 15 Abr 2020]. Disponible en: http://www.mitramiss.gob.es/estadisticas/eat/eat18/ TABLAS ESTADISTICAS/ATR_2018_A.pdf.

2. Cillino S, Casuccio A, Di Pace F, Pillitteri F, Cillino G. A five-year retrospective study of the epidemiological characteristics and visual outcomes of patients hos- 
pitalized for ocular trauma in a Mediterranean area. BMC Ophthalmol. 2008;8:6. doi:10.1186/1471-2415-8-6.

3. Fea A, Bosone A, Rolle T, Grignolo FM. Eye injuries in an Italian urban population: Report of 10620 cases admitted to an eye emergency department in Torino. Graefe's Arch Clin Exp Ophthalmol. 2008;246(2):175-9. doi:10.1007/s00417-0070738-7.

4. Cao H, LL, Zhang M. Epidemiology of Patients Hospitalized for Ocular Trauma in the Chaoshan Region of China, 2001-2010. PLoS One. 2012;7(10):e48377e48377. doi:10.1371/journal.pone.0048377.

5. Ho CK, Yen YL, Chang $\mathrm{CH}$, Chiang HC, Shen YY, Chang PY. Epidemiologic study on work-related eye injuries in Kaohsiung, Taiwan. Kaohsiung J Med Sci. 2007;23(9):463-9. doi:10.1016/S1607-551X(08)70054-8.

6. Cai M, Zhang J. Epidemiological characteristics of work-related ocular trauma in southwest region of China. Int J Environ Res Public Health. 2015;12(8):9864-75. doi:10.3390/ijerph120809864.

7. Sahraravand A, Haavisto AK, Holopainen JM, Leivo T. Ocular traumas in working age adults in Finland - Helsinki Ocular Trauma Study. Acta Ophthalmol. 2017;95(3):288-94. doi:10.1111/aos.13313.

8. Serrano C, Jiménez L, Jerez M, O'Connor S, Bardón I, Caso C. Protección ocular: importancia y uso. Med segur trab. 2008;54(213):81-86. Disponible en: http:// scielo.isciii.es/scielo.php?script=sci_arttext\&pid=S0465-546X2008000400009\&|$\mathrm{ng}=\mathrm{es}$.

9. Gómez P, Ladaria A. Patología oftálmica en el medio laboral: ergooftalmología. Barcelona: Universitat de Barcelona; 2007.

10. Instituto Sindical de Trabajo ambiente y salud (ISTAS). Informe sobre la evolución de la siniestralidad laboral en España. Fundación para la prevención de riesgos laborales. 2015;1-73.

11. Secretaria confederal de salud laboral de CCOO. Evolución de los accidentes de trabajo en España (2012-2018). CC.00. 2019;5-33.

12. Sección prensa / Encuesta de Población Activa (EPA). Serie histórica (Datos en miles de personas) [página principal en Internet]. Madrid: Instituto Nacional de estadística (INE). [actualizado 25 Jan 2021; citado 18 Dec 2019]. Disponible en: https://www.ine.es/prensa/epa_tabla.htm.

13. Edición electrónica de la CIE-9-MC. 9a Edición [página principal en Internet]. Madrid: Ministerio de Sanidad Consumo y Bienestar Social. [actualizado 18 Dec 2020; citado 30 Jul 2019]. Disponible en: https://eciemaps.mscbs.gob.es/ecieMaps/ browser/index_9_mc.html\#search=\&flags=111100\&flags LT=11111111\&searchl$d=1564475344573 \&$ indiceAlfabetico $=\&$ listaTabular $=i d-11528$-class $-360-379 \&$ ex pand $=0 \&$ clasification $=$ cie9mc\&version $=2014$. 
14. WHO | ICD-10 online versions [página principal en Internet]. World Health Organization [actualizado 25 Jan 2021; citado 15 Apr 2020]; Disponible en: https://icd. who.int/browse10/2019/en.

15. Gobba F, Dall'Olio E, Modenese A, De Maria M, Campi L, Cavallini GM. Work-related eye injuries: A relevant health problem. main epidemiological data from a highly-industrialized area of northern Italy. Int J Environ Res Public Health. 2017;14(6):604. doi:10.3390/ijerph14060604.

16. Martin S, Alvarez C, Thuissard I, Catalina C, Calvo E, Villa C, et al. Description of the epidemiological characteristics of work-related eye injuries in Spain. A retrospective study. BMJ Open. 2020;10(10):e035696. doi:10.1136/bmjopen-2019-035696.

17. A. Accidentes de Trabajo con Baja Ocurridos en 2008. A.1.2. Por sector y rama de actividad. Estadísticas de Accidentes de Trabajo. Año 2008. [página principal en Internet]. Madrid: Ministerio de Trabajo Migraciones y Seguridad social. [actualizado 14 Feb 2012; citado 28 Dec 2019]. Disponible en: https://www.mites.gob.es/ estadisticas/eat/eat08/A1/a12_top_HTML.htm.

18. Consejería de Economía empresas y empleo. Estado de la Seguridad y Salud laboral en Castilla La Mancha [edición electrónica] 2017. [actualizado 14 Dec 2017; citado 29 Dec 2019];1-37. Disponible en: https://seguridadlaboral.castillalamancha.es/sites/seguridadlaboral.castillalamancha.es/files/pagina/descargas/estado_situacion_seguridad_y_salud_en_clm.pdf.

19. Departament de treball i indústria $\mathrm{G}$ de $\mathrm{C}$. Plan de Gobierno para la prevención de riesgos laborales 2005-2008 [edición electrónica]. 2006 [actualizado 11 Apr 2007; citado 29 Dec 2019];1-62. Disponible en: https://www.gencat.cat/treball/ doc/doc_21762554_2.pdf.

20. Nolte M. La década perdida. Escritos de un tiempo de crisis (2008-2018). 1st ed. Publicaciones de la Universidad de Deusto: DeustoDigital; 2018. 